\title{
Nanoscale
}

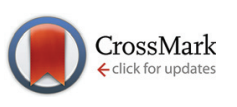

Cite this: Nanoscale, 2017, 9, 2778

\section{Detecting the shape of anisotropic gold nanoparticles in dispersion with single particle extinction and scattering $\dagger$}

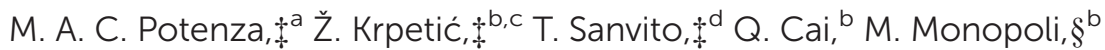 \\ J. M. de Araújo, ${ }^{\text {,e }}$ C. Cella, ${ }^{d}$ L. Boselli, ${ }^{b}$ V. Castagnola, ${ }^{b}$ P. Milani ${ }^{\star a}$ and \\ K. A. Dawson*b
}

\begin{abstract}
The shape and size of nanoparticles are important parameters affecting their biodistribution, bioactivity, and toxicity. The high-throughput characterisation of the nanoparticle shape in dispersion is a fundamental prerequisite for realistic in vitro and in vivo evaluation, however, with routinely available bench-top optical characterisation techniques, it remains a challenging task. Herein, we demonstrate the efficacy of a single particle extinction and scattering (SPES) technique for the in situ detection of the shape of nanoparticles in dispersion, applied to a small library of anisotropic gold particles, with a potential development for in-line detection. The use of SPES paves the way to the routine quantitative analysis of nanoparticles dispersed in biologically relevant fluids, which is of importance for the nanosafety assessment and any in vitro and in vivo administration of nanomaterials.
\end{abstract}

Received 18th November 2016, Accepted 12th January 2017

DOI: $10.1039 / c 6 n r 08977 a$

rsc.li/nanoscale tools and toxicology. ${ }^{3,4}$ The characterisation of nanoparticle interactions in contact with complex biological entities must take into account the behaviour of nanosized objects in different media as the nanoparticles may be affected by a number of biomolecules present therein, which can significantly influence their aggregation state and alter the dispersion quality, overall shape, bioavailability, and efficacy in the case of diagnostic or therapeutic applications. ${ }^{5}$

The physico-chemical properties of nanoparticles influence their toxicological behaviour but the link between the two is far from being completely understood. Due to the high surface-tovolume ratio, the surface of nanoparticles is very reactive and results in complex and dynamic interactions with the surrounding media. ${ }^{6}$ In the case of nanoparticles dispersed in complex biological fluids, a particle low energy state is reached, in some cases, by adsorbing biomolecules from the surrounding medium with the formation of a dynamic/static biomolecular corona. ${ }^{6}$ The adsorption is dependent on the local properties of nanoparticles in dispersion i.e. particle size, core material and surface chemistry which can affect the surface charge, curvature and ultimately the particle shape.$^{7-9}$ Besides surface chemistry, nanoparticle size and shape are key parameters affecting the biological response, i.e. the rate of cellular uptake, biodistribution, bioactivity, therapeutic efficacy and toxicity. ${ }^{10,11}$ This highlights the importance of accurate determination of geometry, morphology and rheology of nanoparticles. ${ }^{12}$

Before using nanoparticle dispersions for biological applications, typically a number of parameters, such as particle size 
and shape distribution, morphology, the stability of dispersions and surface chemistry are determined by conventional characterisation techniques. These include very popular analytical methods such as: dynamic and static light scattering for the determination of NP hydrodynamic radius, size distribution and polydispersity, ${ }^{13}$ atomic force microscopy (AFM) for the analysis of the particle shape, size and distribution along with the surface characterisation, ${ }^{14}$ zeta potential measurements, ${ }^{15}$ UV-Vis spectroscopy for analysis of plasmonic metal nanoparticles (e.g. Au, Ag), ${ }^{16}$ electron microscopy for the analysis of the particle size and shape, ${ }^{17}$ nanoparticle tracking analysis (NTA) ${ }^{18}$ and field flow fractionation (FFF). ${ }^{19}$ Recently, differential centrifugal sedimentation (DCS) allowed high resolution sizing of monolayer protected metal nanoparticles ${ }^{20}$ fine detection of particle aggregation states ${ }^{21}$ and used in addition to other analytical techniques to assess mapping of protein binding at the nanoparticle surface on a particle-by-particle basis. ${ }^{22}$

Most of the above-mentioned techniques are not suitable for in-line, high-throughput nanoparticle analysis since they require off-line manipulation of the dispersion, moreover, do not provide a straightforward characterisation of nanoparticle shapes and/or the evolution for polydisperse samples in fluids. Optical techniques, based on light absorption or scattering, ${ }^{23}$ are, in principle, valid candidates for the direct in situ characterisation of nanoparticles in solution, since they do not require any sample manipulation such as drying, concentration, purification etc.

In spite of being very straightforward and easy to use, dynamic light scattering (DLS) does not provide any information on the nanoparticle structure, shape anisotropy and assembly of the nanoparticle with high polydispersity but provides only the information on the particle hydrodynamic radius. ${ }^{24,25}$

Recently, an approach based on single particle extinction and scattering (SPES) optical characterisation was reported. ${ }^{26-28}$ SPES provides two independent parameters describing the optical field scattered forward from single particles illuminated by a laser beam. These two independent parameters are directly measured from the interference between the transmitted beam and the forward scattered light, thanks to the self-reference interference between the faint scattered and the intensely transmitted fields. ${ }^{27,28}$ The raw data generated by SPES are described with real and imaginary parts of the electric field, Re $S(0)$ and Im $S(0)$ respectively. ${ }^{27,28}$ The former is related to the ability of the particle to scatter and absorb the light and the latter to the capacity of the particle of being polarized under an external field, i.e. polarizability. ${ }^{23}$ Both parameters are influenced by the surface plasmon modes that are being modified upon changes in shape and/or changes in surface features.

This overcomes the limitations typical of DLS, making SPES an efficient tool for the in line characterisation of nanoparticle polydispersity in fluids, providing information on particle size distribution, agglomeration state and refractive index of the dispersed species. ${ }^{26-31}$ In the present configuration, SPES can work with dielectric particles down to roughly $200 \mathrm{~nm}$ in dia-
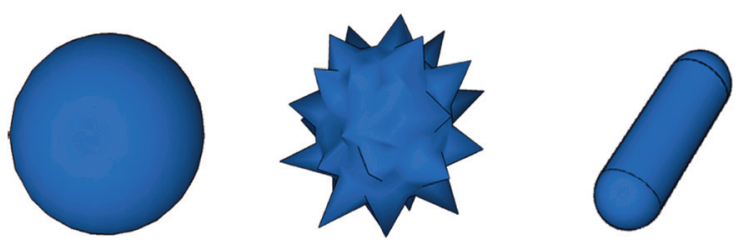

Scheme 1 Schematic representation of the nanoparticle shape library: spherical, branched and rod-shaped nanoparticles.

meter. In the case of metallic NPs, as for example gold, both scattering and extinction efficiencies are much larger than in the case of dielectric particles, increasing the sensitivity of the method for small sizes. Hence, the lower size limit of detection is achieved, i.e. 50-60 $\mathrm{nm}$ for the spherical particles. Moreover, the plasmon properties depend on the particle shape..$^{23,26}$

Herein, we exploit the SPES technique for the challenging characterisation of the particle shape in the case of gold nanoparticles in dispersion, represented with a shape library. As a proof of concept, we use the SPES technique applied on a shape library of gold nanoparticles, consisting of dispersed spherical nanoparticles, branched nanoparticles (nanostars) and nanorods of extremely narrow size distribution (Scheme 1). We demonstrate that SPES allows the quantitative analysis of a range of particle shapes in a solution being able to distinguish between different shapes of particles of comparable size.

We provide experimental evidence that, independently of any previous knowledge of the particles, the SPES method is capable of detecting the surface features of nanoparticles at a scale much smaller than the wavelength of light and therefore well below the limit of optical resolution.

\section{Results and discussion}

Highly monodisperse gold nanoparticles of different shapes were prepared and functionalised with carboxy-PEG thiol ligands to avoid the formation of aggregates. ${ }^{20}$ The shape library of anisotropic gold nanoparticles designed for this work is illustrated in Fig. 1, while full information on the nanoparticle preparation and their physico chemical characterisation is available in the ESI Table S1. $\dagger$ This library consists of nanoparticles with three distinct shapes: spherical gold nanoparticles of 60 and $80 \mathrm{~nm}$ (GNP1, GNP2), branched gold nanoparticles of 60 and $120 \mathrm{~nm}$ nominal core diameter (GNP3, GNP4) and gold nanorods with different aspect ratios $(90 \times 40 \mathrm{~nm} ; 68 \times 13 \mathrm{~nm}$; GNP5, GNP6). We have applied the SPES technique to test its capability to detect different shapes of particles with comparable nominal core diameters.

Note that in order to compare the size range of branched (star-shaped) nanoparticles with spherical nanoparticles we have developed a novel approach to automatically size anisotropic nanoparticles from transmission electron microscopy (TEM) micrographs. Whilst geometrical identification and hence the size distribution of spherical nanoparticles from 


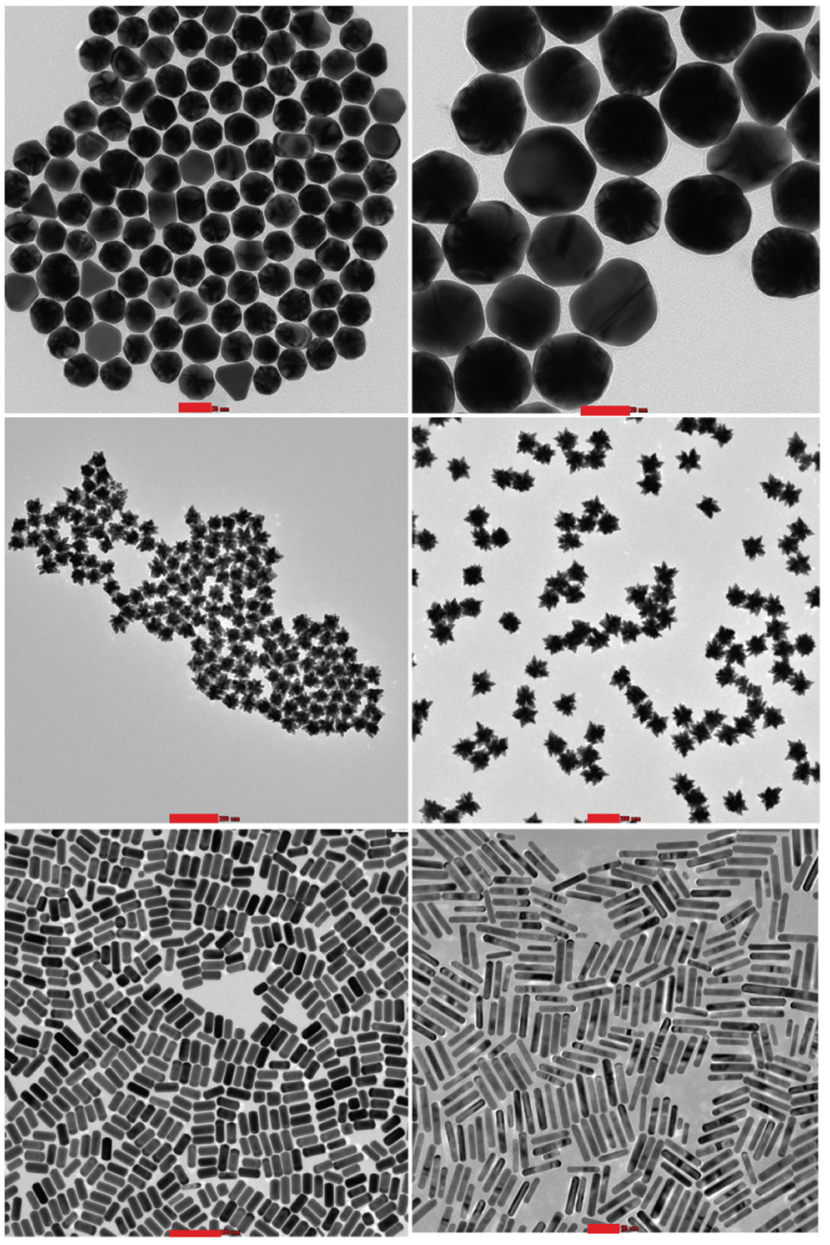

Fig. 1 Gold nanoparticle shape library: representative TEM micrographs of nanoparticles used in this study, i.e. spherical gold nanoparticles (GNP1, GNP2), branched gold nanoparticles (GNP3, GNP4) and gold nanorods (GNP5. GNP6). Scale bars are 50, 50, 200, 200, 200 and $50 \mathrm{~nm}$, respectively.

TEM micrographs can be easily obtained using automatic software approaches (e.g. ImageJ software), determining the size distribution of branched particles is not a trivial task. ${ }^{17}$ We developed a software specifically aimed to identify and process nanoparticles using TEM micrographs in a semiautomatic fashion. The idea was to use "ingredients" from different theories (percolation, cluster analysis, heuristic algorithms) in order to automatically identify the nanoparticles. The details of this approach are available in the ESI. $\dagger$

SPES analysis was performed by flowing aqueous dispersions of gold NPs through a laser beam at a constant, known speed and the signals from the detectors were sampled at a frequency higher than the inverse transit time to accurately follow in time the self-reference interference fringes. As an output, the SPES produces two-dimensional (2D) plots (histograms) providing the raw data distribution of single particle fields in the complex plane Re $\mathrm{S}(0)-\operatorname{Im~S}(0) .{ }^{27}$ Typically, the scattered field depends on (i) size, (ii) refractive index and (iii) shape and orientation of each particle.
Fig. 2 shows how spherical (GNP2) and branched gold nanoparticles (GNP3, GNP4) in a comparable size range occupy different regions of the two-dimensional plot. The SPES raw data, represented in 2D histograms, show the number of particles measured within each $2 \mathrm{D}$ bin with grey tones i.e. the darker the grey tone, the larger the number of particles. The resulting analysis of spherical gold particles of $80 \mathrm{~nm}$ in diameter and two samples of branched particles (60 and $124 \mathrm{~nm}$ nominal diameter) clearly indicate the capability of the system to distinguish between them independently of any information about the particles, observing a large shift in the case of branched particles towards larger real and smaller imaginary values. Moreover, it also shows an estimate of the actual resolution of the SPES technique, considering that we are characterising highly monodisperse samples. The data spread in the $2 \mathrm{D}$ plot provides the resolution of the experimental set-up used here according to fundamental definitions of resolution. ${ }^{32}$ The spread is still smaller than the separation between the data sets. We have not used here more refined algorithms like deconvolution or any inversion process to analyze data. These approaches are nevertheless absolutely viable and easy to be implemented if necessary, as it occurs for any resolution limited measuring device. We limit ourselves to evaluate the centroid of the data distributions, which results in the most probable value based on the statistical analysis and is easily comparable with the theoretical expectations.

The rationale of the shift observed in Fig. 2 is explained via a semi-quantitative interpretation based on the Drude simplified model detailed in the ESI. $\dagger^{23}$ By considering the scattered

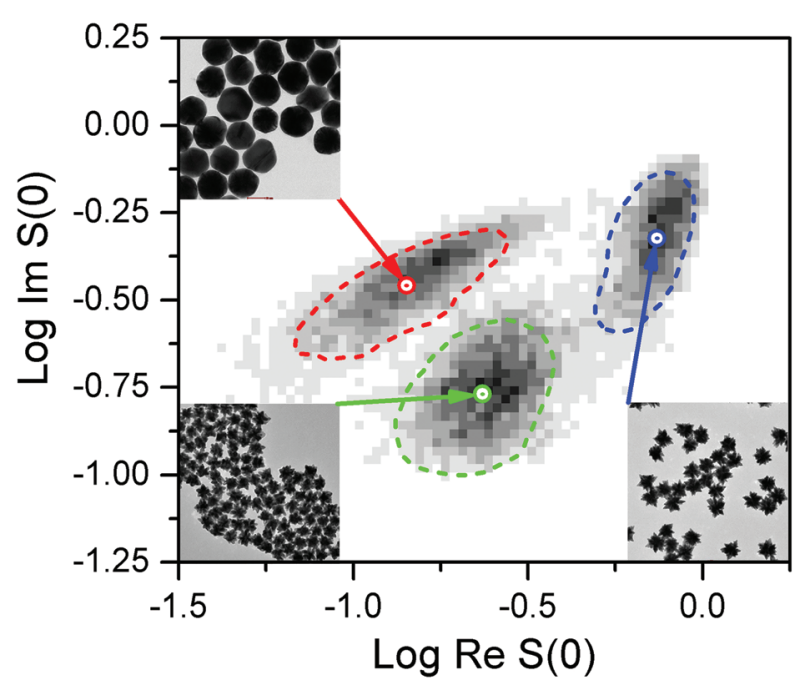

Fig. 2 SPES raw data representative for spherical (GNP2, red) and branched gold nanoparticles (GNP3, green; GNP4, blue) with the corresponding centroids indicated. Comparing the results obtained for GNP2 and GNP3, the effect of the charge of the surface plasmon is evidenced by the strong shift (almost a decade) in the horizontal direction. Raw data for the forward scattered field amplitudes are represented here, as described in the text. Grey tones indicate on a linear scale the number of particles within each bin. In the insets, the corresponding TEM micrographs of the nanoparticles are shown. 
field $S(0)$ at the first order of the expansion, and thanks to the simple assumption about the influence of surface corrugation of the plasmon resonance (see the ESI $\dagger$ ), we expect a stronger absorption and weaker scattering for branched particles compared to spherical nanoparticles, as shown in Fig. 2.

Fig. 3 reports the results obtained for nanospheres (a, b) and nanorods (c, d). Data are presented separately for two reasons: (i) the samples have been measured separately; (ii) some data sets are partially superimposed, thus making it difficult to compare the corresponding SPES raw data.

By exploiting basic statistical approaches it is possible to deconvolute the position of the centroids, even in the cases of overlapping data distributions. This approach is not the purpose of the present work, and therefore we only extract the centroids of each data set. A clear systematic shift is evident between the spherical and rod-shaped nanoparticles when comparing the position of the data sets in the $2 \mathrm{D}$ plane when the separation between centroids becomes excessive. Herein we exploit the estimate of the instrumental resolution mentioned above. Even in the case of Fig. 3(b) and (d), the separation of the centroids is at least comparable to the width of the data sets (evidenced by the dashed lines). This result shows that basic resolution criteria like the Rayleigh's one for spectral lines are enough to separate spheres from rods even if data are partially superimposed. ${ }^{32}$

We highlight that these assessments are solely based on the experimental data provided, in an absolutely model-independent way. The Drude simplified model (see the ESI $\dagger$ ) can be used to explain the differences between the results reported in Fig. 3. Both size and shape can be identified without any free parameter for nanorods of different aspect ratios. For simplicity, we approximate the nanorods as ellipsoids. Under the small particle approximation, the polarizability for an ellipsoid can be obtained. ${ }^{23}$ As shown in the ESI, $\dagger$ an accurate evaluation of polarizability and scattered fields for ellipsoids is then possible without any free parameter by introducing the same
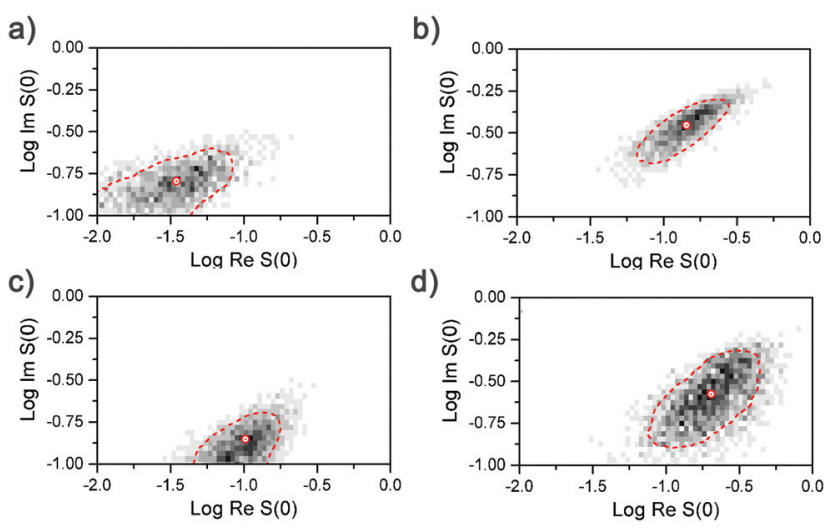

Fig. 3 SPES raw data for (a) GNP1, (b) GNP2 (spherical gold nanoparticles), (c) GNP6, and (d) GNP5 (gold nanorods). The centroids of the 2D distributions are indicated with crosses. The effect of shape is again to give appreciable horizontal shift, showing the change of polarizability induced by the change in the surface plasmons. properties used for the description of spheres. The additional degree of freedom given by the particle orientation must be taken into account due to the non-spherical shapes. This makes the polarizability and the fields, both real and imaginary parts, to change by almost an order of magnitude upon orientation. This is not evident from the results in Fig. 3(c and d), where the data spread is appreciably smaller, still comparable to the estimated resolution. In fact, we evaluated the characteristic time constants for the Brownian rotations of these nanoparticles to be smaller than the time needed to traverse the laser beam in the focal plane. Therefore, any particle generates an optical signal, which is an average of the orientations assumed during the passage through the beam (see Analytical methods). As a consequence, the fast orientation changes are responsible for the relatively reduced spread of the population observed in Fig. 3 with respect to all possible values. This causes the final results exhibiting a limited uncertainty, which allows for a very good separation of (small) rods from spheres.

\section{Experimental methods}

\section{Chemicals}

All the reagents used in this work were of the highest available grade and were purchased from Sigma Aldrich, if not stated otherwise. Hydrogen tetrachloroaurate trihydrate ( $\geq 99.9 \%)$, trisodium citrate trihydrate, sodium borohydride ( $\geq 99 \%)$, bis( $p$-sulfonatophenyl)phenylphosphine dihydrate dipotassium salt (BSSP, 97\%), hexadecyltrimethylammonium bromide (CTAB, $\geq 99 \%$ ), hexadecyltrimethylammonium chloride (CTAC, $\geq 98 \%$ ), L-ascorbic acid ( $\geq 99 \%)$, potassium carbonate ( $\geq 99 \%)$, tannic acid, silver nitrate ( $\geq 99.9 \%)$, hydrochloric acid $(37 \%$, AR grade). For the preparation of nanoparticles, HPLC grade purity water was purchased from Sigma Aldrich, namely LCMS Ultra CHROMASOLV®). Sodium oleate (NaOL, $\geq 97 \%$ ) was purchased from Tokyo Chemical Industry Co., Ltd and a carboxy-PEG thiol ligand ( $\left.\mathrm{HS}-\mathrm{C}_{11}-\mathrm{EG}_{6}-\mathrm{OCH}_{2}-\mathrm{COOH}\right)$ from ProChimia Surfaces.

\section{Preparation of gold nanoparticles}

Spherical gold nanoparticles. Gold nanoparticles of 60 and $80 \mathrm{~nm}$ nominal core diameters chosen for this work were purchased from BBI Solutions. The particles were functionalised with a carboxy-PEG thiol ligand (ProChimia) HS- $\left(\mathrm{CH}_{2}\right)_{11}-[\mathrm{EG}]_{6}-$ $\mathrm{OCH}_{2}-\mathrm{COOH}$ (referred as PEG6-COOH further in the text). The surface ligand provides stability against aggregation and excellent dispersion in aqueous media.

For the surface functionalisation, the as received gold colloid was placed in six glass vials $(5 \mathrm{~mL}$ per vial) and subsequently, $20 \mu \mathrm{L}$ of the aqueous solution of the functional PEG-ligand $^{33}(50 \mu \mathrm{M})$ was added under vigorous stirring to each vial $(30 \mathrm{~s})$. The mixtures were then left overnight and subsequently excess ligands were removed by repetitive cycle centrifugation (5 min, 4000/5000 rpm, $10^{\circ} \mathrm{C}$ ) and redispersed 
in fresh milli-Q water (resistivity $8.2 \mathrm{M} \Omega \mathrm{cm}$ at $25{ }^{\circ} \mathrm{C}$ ). The final volume of the colloidal dispersion was $1 \mathrm{~mL}$.

Gold nanorods. Gold nanorods were prepared using a seeded-growth method reported in the literature. ${ }^{34,35}$

Step 1. Seed preparation. $5 \mathrm{~mL}$ of $0.5 \mathrm{mM}$ of $\mathrm{HAuCl}_{4}$ was added to $5 \mathrm{~mL}$ of $0.2 \mathrm{M}$ of $\mathrm{CTAB}$ in a glass vial under vigorous stirring, followed by the addition of $1 \mathrm{~mL}$ of freshly prepared $6 \mathrm{mM} \mathrm{NaBH}_{4}$ under vigorous stirring. The mixture was vigorously stirred for $2 \mathrm{~min}$ and aged at room temperature for a further $30 \mathrm{~min}$. The colour of the colloidal dispersion resulted light brown. Prepared seeds were utilised within 1 day of preparation.

Step 2. Gold nanorod preparation. Growth solution was prepared as follows: the required amount of CTAC/CTAB and sodium oleate (NaOL) (see Table $\mathrm{S} 2 \uparrow$ for details on quantities) was dissolved in $250 \mathrm{~mL}$ of warm milli-Q water $\left(40-50^{\circ} \mathrm{C}\right)$, in a $1 \mathrm{~L}$ round bottom flask, then the solution was kept at $30^{\circ} \mathrm{C}$ in an oil bath. Subsequently, a certain volume of $\mathrm{AgNO}_{3}(4 \mathrm{mM})$ was added into the flask (see Table $\mathrm{S} 2 \dagger$ ) and the mixture was kept undisturbed for $15 \mathrm{~min}$ at $30^{\circ} \mathrm{C}$. Then $250 \mathrm{~mL}$ of $\mathrm{HAuCl}_{4}$ $(1 \mathrm{mM})$ was added into the flask under stirring $(700 \mathrm{rpm})$, the colour of the mixture changed from yellow to light yellow in 3 h. A certain volume of $\mathrm{HCl}(37 \mathrm{wt} \%$ in water) (see Table $\mathrm{S} 2 \dagger$ for details) was then added under stirring (400 rpm, $15 \mathrm{~min}$ ) followed by the addition of $1.25 \mathrm{~mL}$ aqueous ascorbic acid (64 mM) under vigorous stirring (1200 rpm, $30 \mathrm{~s})$. The colour of the mixture changed from yellow to colourless following the reduction of $\mathrm{Au}(\mathrm{III})$ to $\mathrm{Au}(\mathrm{I})$. Subsequently, a required volume of gold seeds was added under vigorous stirring (1200 rpm, $30 \mathrm{~s}$ ) and the dispersion was kept undisturbed at $30{ }^{\circ} \mathrm{C}$ overnight until the completion of the reaction. The resulting colloidal dispersion was filtered through $0.2 \mu \mathrm{m}$ Millipore ${ }^{\circledR}$ syringe filters before surface modification.

For the surface modification, $20 \mu \mathrm{L}$ of aqueous functional PEG ligand $(50 \mu \mathrm{M})$ was added under stirring to $20 \mathrm{~mL}$ of the gold nanorod (GNR) dispersion. The mixtures were then left overnight and subsequently excess ligands were removed by repetitive cycle centrifugation ( $5 \mathrm{~min}, 4000 / 5000 \mathrm{rpm}, 25^{\circ} \mathrm{C}$ ) and redispersed in fresh milli-Q water. The final volume was 1-5 mL, depending on the sample.

Branched gold nanoparticles (gold nanostars). Branched gold particles were prepared using a two-step seeded growth modified literature method. ${ }^{36}$

Step 1. Seed preparation. Gold seeds of ca. $10 \mathrm{~nm}$ diameter were prepared by citrate reduction of $\mathrm{HAuCl}_{4}$, i.e. a modified Turkevich-Frens method. ${ }^{20,37,38}$ Briefly, $900 \mu \mathrm{L}$ of $\mathrm{HAuCl}_{4}$ ( $1 \mathrm{wt} \%$ ) were added to $90 \mathrm{~mL}$ of milli-Q water and heated to boiling under reflux. Subsequently, $2.7 \mathrm{~mL}$ of trisodium citrate (1 wt\%) were quickly injected into the mixture under vigorous stirring. The mixture was left under reflux for $10 \mathrm{~min}$. After the formation of the ruby red colour, the flask was placed on ice and allowed to cool to room temperature. Subsequently, the particles were filtered through a $0.2 \mu \mathrm{m}$ Millipore ${ }^{\circledR}$ filter and kept at $4{ }^{\circ} \mathrm{C}$ up to two weeks before use.

Step 2. Formation of branches and particle growth. Different sizes of branched gold nanoparticles were prepared via the seeded growth method using the spherical gold seeds prepared as described in step 1. In a typical preparation, $96 \mathrm{~mL}$ of milli$\mathrm{Q}$ water was poured into a $250 \mathrm{~mL}$ beaker tall form equipped with a magnetic stir bar to allow high vortex during the reaction. To this $100 \mu \mathrm{L}$ of $\mathrm{HAuCl}_{4}(100 \mathrm{mM})$ was added, followed by an addition of $500 \mu \mathrm{L}$ of gold seeds, $250 \mu \mathrm{L}$ of trisodium citrate $(1 \mathrm{wt} \%)$ and $250 \mu \mathrm{L}$ of freshly prepared aqueous hydroquinone $(0.14 \mathrm{M})$. The mixture was vigorously stirred for 10 min allowing the formation of branched particles (a cerulean blue coloured gold colloid was observed). In order to tune the overall size of the branched nanoparticles, the amount of gold seeds added to the reaction mixture was changed (see Table S3†).

To the obtained dispersions of branched gold nanoparticles, $1 \mathrm{~mL}$ of aqueous $\operatorname{bis}(p$-sulfonatophenyl)phenylphosphine dihydrate dipotassium (BSPP) ${ }^{39}$ (75 $\mathrm{mM}$ ) was added and stirred for $30 \mathrm{~s}$, to quench the branch growth reaction. Subsequently, for the ligand exchange/functionalisation, $20 \mu \mathrm{L}$ of the aqueous solution of the functional-PEG thiol ligand $(50 \mu \mathrm{M})$ was added under stirring to each vial. The mixtures were then left overnight and subsequently washed by repetitive cycle centrifugation ( $5 \mathrm{~min}, 4000-5000 \mathrm{rpm}, 25^{\circ} \mathrm{C}$ ) redispersing in fresh milli-Q water to a final volume of $1 \mathrm{~mL}$.

\section{Analytical methods}

Single particle extinction and scattering (SPES). The complex amplitude of the scattered field has been measured through a SPES device described in previous studies. ${ }^{27,28}$ A $5 \mathrm{~mW}$ solid-state laser (wavelength $635 \mathrm{~nm}$ ) has been focused to a diameter of $5 \mu \mathrm{m}$ into a flow cell and the emerging light collected by a segmented photodiode. A small particle passing through the focal region generates a scattered wave that superimposes to the intensely transmitted beam. Aqueous dispersions of particles were passed through the beam at a constant speed of $0.1 \mathrm{~m} \mathrm{~s}^{-1}$ and the electric signals from the sensors were sampled at a frequency of $1 \mathrm{MHz}$, higher than the inverse transit time. Therefore a detailed history of the signals was obtained and passed through a dedicated pulse shape analyser to extract the field amplitude of each particle. The method automatically provides an intrinsic calibration of the signals, thus allowing the data interpretation without any free parameter.

SPES is advantageous when working with metallic nanoparticles, in particular, those made of gold. Thanks to the plasmonic properties of gold nanoparticles, both scattering, and extinction efficiencies are much larger than in the case of dielectric particles, increasing the sensitivity of the method for small sizes. Moreover, the surface plasmons are strongly affected by size and shape, largely increasing the sensitivity in distinguishing different sizes and shapes (ESI $\dagger$ ).

UV-Visible spectroscopy. UV-Vis spectra were recorded on a Varian's Cary®6000i spectrophotometer, registering the spectra in the 400-800 nm range (for spherical gold nanoparticles and nanorods) and 400-1400 nm range (for branched gold nanoparticles) using a quartz cuvette with a path length of $1 \mathrm{~cm}$. 
Differential centrifugal sedimentation (DCS). Monodispersity and particle size distributions were analysed using a disc centrifuge DC24000 (CPS Instruments Inc.). For analysis, the disc was filled with sucrose-based density gradients $(8-24 \mathrm{w} / \mathrm{w} \%)$ using freshly prepared aqueous solutions in Milli-Q and filled successively in nine consecutive steps into the disc (rotating at a maximum speed of $24000 \mathrm{rpm}$ ) starting with the solution of the highest density. ${ }^{20}$ Subsequently, $0.5 \mathrm{~mL}$ of dodecane was injected into the gradient, as a gradient capping agent, to prevent any evaporation of aqueous gradient fluid due to any heating of the disc occurring during analysis. Calibration was performed using a polyvinylchloride (PVC) standard $(0.476 \mu \mathrm{m}$, Analytik Ltd). Calibration was carried out before each measurement by injecting $0.1 \mathrm{~mL}$ of the standard. Similarly, for analysis $0.1 \mathrm{~mL}$ of each sample diluted to an appropriate sample concentration was injected into the disc. Note that in the case of nanorods, a non-sphericity factor of 0.5 was set by the instrument software. However, the diameter/aspect ratio of gold nanorods reported herein was determined using TEM.

Transmission electron microscopy (TEM). Transmission electron microscopy (TEM) micrographs of gold nanoparticles used in this work were acquired using an $\mathrm{FEI}^{\mathrm{TM}}$ Tecnai $^{\mathrm{TM}} \mathrm{G} 2$ 20 Twin microscope (FEI, Inc.). The microscope is equipped with a thermionic LaB6 cathode instrument operating at an accelerating voltage of $200 \mathrm{kV}$. Samples were prepared by evaporating $c a .10 \mu \mathrm{L}$ of appropriately diluted nanoparticle dispersions onto Formvar/carbon-coated copper grids (Agar Scientific; 400 mesh; square).

Nanoparticle tracking analysis (NTA). The number concentration of the samples was measured with a Nanosight LM10 Nanoparticle Analysis System equipped with the NTA 1.3 Analytical Software (Nanosight Ltd). The sample chamber was cleaned with water and ethanol before starting measurements. Approximately $1 \mathrm{~mL}$ of the sample was loaded into the chamber using a disposable syringe. A video of the particle movement, i.e. Brownian motion was recorded for each measurement (30 seconds long). Milli-Q water was used as the dispersant in all measurements. The temperature was detected with a temperature probe before analysing the video record in order to adjust the viscosity of the solution.

Dynamic light scattering (DLS). Particle size distributions were also measured using a Zetasizer Nano ZS (Malvern Instruments Ltd). A detection angle of $173^{\circ}$ and a detection temperature of $25{ }^{\circ} \mathrm{C}$ were applied to all measurements. The light source was a $4 \mathrm{~mW}$ He-Ne laser with a red $(633 \mathrm{~nm})$ wavelength. $1.5 \mathrm{~mL}$ PMMA disposable cuvettes (BRAND, Cat. no. 759115) were used, and approximately $0.8 \mathrm{~mL}$ of the sample was used to fill each cuvette. Milli-Q Water was used as the dispersant in all cases. For each measurement, 3 measurement runs were performed, having 12-16 sub-runs. The particle refractive index was set to 0.47 (gold) while the dispersant refractive index was set to 1.33 (water).

\section{Conclusions}

In conclusion, we demonstrated the capability of the SPES technique to distinguish between different shapes of gold nanoparticles in dispersion and its sensitivity to the surface features in the case of particles of comparable hydrodynamic size.

This approach solves a big challenge and brings the potential for routine screening of different shapes of nanoparticles in dispersion, without necessarily utilising off-line sophisticated and expensive tools based on the electron or scanning probe microscopies, and without the need for laborious analysis using off-line techniques. The major breakthrough of SPES consists of the short analysis time (a few minutes), precision of output and applications to in-line analysis approaches, making this tool a valuable support for the in vitro and in vivo evaluation of the effects of nanoparticle shapes and for optimisation of the large-scale production processes of nanoparticles with controlled shapes.

\section{Acknowledgements}

Ž. K., P. M. and K. D. acknowledge the financial support of the EU FP7 FutureNanoNeeds project (NMP.2013.1.3-3) under the grant agreement number 604602. J. M. A. acknowledges CNPq - Conselho Nacional de Desenvolvimento Científico e Tecnológico, of the Ministry of Science, Technology and Innovation of Brazil.

\section{References}

1 E. J. Cho, H. Holback, K. C. Liu, S. A. Abouelmagd, J. Park and Y. Yeo, Mol. Pharmaceutics, 2013, 10, 2093.

2 A. Lopez-Serrano, R. M. Olivas, J. S. Landaluze and C. Camara, Anal. Methods, 2014, 6, 38.

3 M. P. Monopoli, D. Walczyk, A. Campbell, G. Elia, I. Lynch, F. Baldelli Bombelli and K. A. Dawson, J. Am. Chem. Soc., 2011, 133, 2525.

4 K. P. Donaldson and C. A. Poland, Curr. Opin. Biotechnol., 2013, 24, 724.

5 A. Albanese and W. C. W. Chan, ACS Nano, 2011, 5, 5478.

6 Ž. Krpetić, S. Anguissola, D. Garry, P. Kelly and K. Dawson, in Nanomaterial: Impacts on Cell Biology and Medicine, ed. D. G. Capco and Y. Chen, Springer, Netherlands, 2014, 135-156.

7 S. Mirsadeghi, R. Dinarvand, M. H. Ghahremani, M. R. Hormozi-Nezhad, Z. Mahmoudi, M. J. Hajipour, F. Atyabi, M. Ghavami and M. Mahmoudi, Nanoscale, 2015, 7, 5004.

8 W. Pengyang, W. Xin, W. Liming, H. Xiaoyang, L. Wei and C. Chunying, Sci. Technol. Adv. Mater., 2015, 16, 034610.

9 A. Bigdeli, S. Palchetti, D. Pozzi, M. R. Hormozi-Nezhad, F. Baldelli Bombelli, G. Caracciolo and M. Mahmoudi, ACS Nano, 2016, 10, 3723. 
10 A. Albanese, P. S. Tang and W. C. W. Chan, Annu. Rev. Biomed. Eng., 2012, 14, 1.

11 Y. Li, M. Kroger and W. K. Liu, Nanoscale, 2015, 7, 16631.

12 R. Toy, P. M. Peiris, K. B. Ghaghada and E. Karathanasis, Nanomedicine, 2014, 9, 121.

13 R. Xu, Particle Characterization: Light Scattering Methods, Springer, Netherlands, London, 2000.

14 C. M. Hoo, N. Starostin, P. West and M. L. Mecartney, J. Nanopart. Res., 2008, 10, 89.

15 N. Arjmandi, W. Van Roy, L. Lagae and G. Borghs, Anal. Chem., 2012, 84, 8490.

16 W. Haiss, N. T. K. Thanh, J. Aveyard and D. G. Fernig, Anal. Chem., 2007, 79, 4215.

17 T. Allen, Particle Size Measurement, Springer, Netherlands, 1997.

18 V. Filipe, A. Hawe and W. Jiskoot, Pharm. Res., 2010, 27, 796.

19 M. Baalousha, B. Stolpe and J. R. Lead, J. Chromatogr. A, 2011, 1218, 4078.

20 Ž. Krpetić, A. M. Davidson, M. Volk, R. Levy, M. Brust and A. I. Cooper, ACS Nano, 2013, 7, 8881.

21 Ž. Krpetić, I. Singh, W. Su, L. Guerrini, K. Faulds, G. A. Burley and D. Graham, J. Am. Chem. Soc., 2012, 134, 8356.

22 P. M. Kelly, C. Åberg, E. Polo, A. O’Connell, J. Cookman, J. Fallon, Ž. Krpetić and K. A. Dawson, Nat. Nanotechnol., 2015, 10, 472.

23 C. F. Bohren and D. R. Huffman, Absorption and Scattering of Light by Small Particles, Wiley-VCH Verlag GmbH, 2007, 57-81.
24 W. I. Goldburg, Am. J. Phys., 1999, 67, 1152.

25 B. J. Frisken, Appl. Opt., 2001, 40, 4087.

26 M. A. C. Potenza and P. Milani, J. Nanopart. Res., 2014, 16, 1.

27 M. A. C. Potenza, T. Sanvito and A. Pullia, AIP Adv., 2015, 5, 117222.

28 M. A. C. Potenza, T. Sanvito, S. Argentiere, C. Cella, B. Paroli, C. Lenardi and P. Milani, Sci. Rep., 2015, 5, 18228.

29 M. A. C. Potenza, K. P. V. Sabareesh, M. Carpineti, M. D. Alaimo and M. Giglio, Phys. Rev. Lett., 2010, 105, 193901.

30 M. A. C. Potenza, T. Sanvito and A. Pullia, J. Nanopart. Res., $2015,17,1$.

31 S. Villa, T. Sanvito, B. Paroli, A. Pullia, B. Delmonte and M. A. C. Potenza, J. Appl. Phys., 2016, 119, 224901.

32 J. W. Goodman, Introduction to Fourier Optics, McGraw Hill, 1996.

33 A. G. Kanaras, F. S. Kamounah, K. Schaumburg, C. J. Kiely and M. Brust, Chem. Commun., 2002, 2294.

34 X. Ye, Y. Gao, J. Chen, D. C. Reifsnyder, C. Zheng and C. B. Murray, Nano Lett., 2013, 13, 2163.

35 X. Ye, C. Zheng, J. Chen, Y. Gao and C. B. Murray, Nano Lett., 2013, 13, 765.

36 J. Li, J. Wu, X. Zhang, Y. Liu, D. Zhou, H. Sun, H. Zhang and B. Yang, J. Phys. Chem. C, 2011, 115, 3630.

37 J. Turkevich, P. C. Stevenson and J. Hillier, Discuss. Faraday Soc., 1951, 11, 55.

38 G. Frens, Nat. Phys. Sci., 1973, 241, 20.

39 C. J. Loweth, W. B. Caldwell, X. G. Peng, A. P. Alivisatos and P. G. Schultz, Angew. Chem., Int. Ed., 1999, 38, 1808. 